\title{
Discretization AND ANALYSIS OF THE EVAluATiOn METHOD FOR DESIGN REPORTS
}

\author{
Andrew Sowinski \\ Department of Chemical and Biological Engineering, University of Ottawa, Ottawa, ON, Canada, K1N 6N5 \\ sowinski@uottawa.ca
}

\begin{abstract}
A fair and consistent evaluation of project reports is one of the most difficult tasks in a course. Even with the use of rubrics, subjectivity is still inherently dominant when assigning a level to each rubric criterion and can even vary between evaluators. By coding and commenting on each report, it is possible to discretize the evaluation into smaller components and to effectively quantify the errors. This would increase the objectification and fairness in the evaluation. This work focuses on the preliminary comparison of using a standard rubric, with analysis of report comments from 21 group reports of a $4^{\text {th }}$ year chemical engineering course. It was found that there was some correlation between the final overall mark assigned through the rubric and the average number of errors per page. The discretization of comments into a quantifiable measure is a promising method to provide a more objective marking scheme.
\end{abstract}

Keywords: Design project rubrics, objective evaluation, data analysis

\section{INTRODUCTION}

Evaluations, although ubiquitous and vital to all engineering courses, are always at the centre of debate due to consistency and fairness of marking. Design courses with a written report component have even more challenges to remain as objective as possible. One method for creating a less subjective and more transparent evaluation for a written report is through the use of rubrics. Rubrics have been shown to promote and improve instruction with clear expectations and criteria, while simultaneously being able to provide feedback and self-direction [1]. However, rubrics have also been shown to be effective only if the rater has been appropriately trained on the design and use of rubrics [2]. They have also shown to promote higher learning strategies for users compared to when a rubric was not used, but also causes issues of coping with stress during the evaluation [3].
Similarly, in a review study by Panadero and Jonsson [4], it was found that rubric are effective, but some factors such as goal orientation and prior academic performance of a student may have effect on current performance. With a rubric, are students simply following the rubric for grades and becoming less creative? Rubrics are useful for setting the expectations, but sometimes not all aspects of quality can be explicitly written into a rubric $[5,6]$.

With group reports, there are often a large variation in the quality of the written reports, making it difficult to accurately assess a particular section of the report, and may again rely more on a subjective evaluation. Subjectivity could also be an issue when there are multiple evaluators using the same rubric. This work aims at analyzing the mechanics of a course by breaking down a report into discrete comments and evaluating the frequency and severity of each comment. These results are then compared to a preset marking rubric to determine if there is any pattern in the evaluation. If objective commenting can be used as a fair method evaluation, then the evaluation becomes less evaluator dependent. Evaluators would only need to evaluate the errors, then the errors could be tabulated and help assign a mark.

\section{METHODOLOGY}

This work focused on a $4^{\text {th }}$ year chemical engineering laboratory course. There were 21 groups with four to five students per group. Each group performed two experiments over the course of the semester. At the end of each experiment students needed to submit a technical written report and were evaluated on five generic report sections:

1. Front Matter (e.g., abstract)

2. Background \& Design

3. Results \& Discussion

4. Conclusions

5. Appendices (e.g., sample calculations)

In each of these sections, the reports were evaluated on seven different competencies. The competencies and brief list of questions used to evaluate them is listed below: 
1. Completeness - Is the section complete and not missing any detail? Is it sufficiently thorough and providing all the required information?

2. Technical Knowledge - Does the section use sound technical knowledge studied in class and/or derived from other reputable resources? Is the knowledge completely accurate?

3. Structure \& Clarity - Does the section follow a logical order and is easy to follow?

4. Conciseness - Is the section concise and does not repeat or provide unnecessary information?

5. Convincingness - Does the section effectively convince the reader its main points are true and valid?

6. Spelling \& Grammar - Is the section essentially free of spelling and grammatical errors?

7. Presentation - Are the table and figures professionally produced, needed, and help in illustrating a point in the section? Are there any major issues with formatting, spacing etc.?

To evaluate the intersection of each competency and section (e.g., Technical Knowledge, in the Results \& Discussion section) a non-linear six-level scale was employed. The scale and its assigned weighted value are shown in Table 1.

Table 1: The marking scale for the evaluation.

\begin{tabular}{|l|l|}
\hline Category & Weight \\
\hline Unsatisfactory & $0 \%$ \\
\hline Poor & $30 \%$ \\
\hline Marginal & $55 \%$ \\
\hline Good & $75 \%$ \\
\hline Very Good & $85 \%$ \\
\hline Excellent & $100 \%$ \\
\hline
\end{tabular}

An example of completed rubric can be seen in Figure 1. The marks were assigned after the commenting was completed, but prior to being analyzed. Each section and competency had their own an associated weight. The value for each intersection of section and competency, (i.e., a cell) was equal to the product of the marking scale (e.g., Excellent, Very Good, et.c), the weight of the section and the weight of the competency. This allowed for a breakdown of the mark per section over all competencies (i.e., the weighted sum of a row), as well as each competency over the entire report (i.e., the weighted sum of the column). Finally, all cells could be weighted to determine the overall report mark.

The reports were submitted electronically and commented using Adobe Acrobat Pro. Each comment contained a coded text that had four components as follows:

\section{SEC $|\mathrm{COMP}| \mathrm{SEV} \mid \mathrm{COM}$}

where SEC, COMP, SEV, and COM represent the section of the comment in the report, the competency being measured, the severity of the issue, and specific comment to the issue, respectively. The severity was evaluated as a major error (i.e., fundamental error), minor error, general comment, excellent work (i.e., above expectations). Once completed, the comments were exported to an Adobe Acrobat xml format, .xrdf, and subsequently transformed into useable tabular data. Other quantitative measures were also collected for each report such as the number of pages, words, figures and tables per section. With the data collected, it is possible to compare the consistency between the number of errors and the overall mark.

\section{RESULTS AND DISCUSSION}

All 21 reports were analyzed with the same methodology. For these reports, the appendix was marked separately by teaching assistants, and therefore this analysis will not consider the grades associated with the appendix. The report did not have any limitation on the number of pages, which lead to large range of submitted reports. The report descriptors can be seen in Figure 2. On

\begin{tabular}{|c|c|c|c|c|c|c|c|c|c|}
\hline \multicolumn{10}{|c|}{ Report } \\
\hline & & Completeness & $\begin{array}{c}\text { Technical } \\
\text { Knowledge }\end{array}$ & $\begin{array}{c}\text { Structure \& } \\
\text { Clarity }\end{array}$ & Conciseness & Convincingness & $\begin{array}{c}\text { Spelling \& } \\
\text { Grammar }\end{array}$ & Presentation & Overall \\
\hline Section & Weight & 25 & 25 & 20 & 10 & 10 & 5 & 5 & 100 \\
\hline Front Matter & 5 & Very Good & Outstanding & Outstanding & Very Good & Outstanding & Very Good & Outstanding & $94.0 \%$ \\
\hline Background \& Design & 25 & Very Good & Very Good & Very Good & Good & Good & Very Good & Good & $82.5 \%$ \\
\hline Results \& Discussion & 40 & Very Good & Very Good & Good & Good & Very Good & Very Good & Good & $81.5 \%$ \\
\hline Conclusions & 5 & Very Good & Very Good & Outstanding & Outstanding & Very Good & Outstanding & Outstanding & $91.0 \%$ \\
\hline Appendix & 25 & Very Good & Very Good & Very Good & Very Good & Very Good & Outstanding & Very Good & $85.8 \%$ \\
\hline Overall & 100 & $85.0 \%$ & $85.8 \%$ & $82.5 \%$ & $79.3 \%$ & $83.3 \%$ & $89.5 \%$ & $80.0 \%$ & $83.9 \%$ \\
\hline
\end{tabular}

Fig. 1. Example of the rubric used to evaluate the lab reports 
average there were approximately 40 pages, 7500 words, and 20 Tables and Figures per report; however, there were reports with more than 15000 words showing a wide range of variability. Generally, there were approximately 150 comments written in each report, but again there was a large variability ranging between 50 and 250 comments per report.
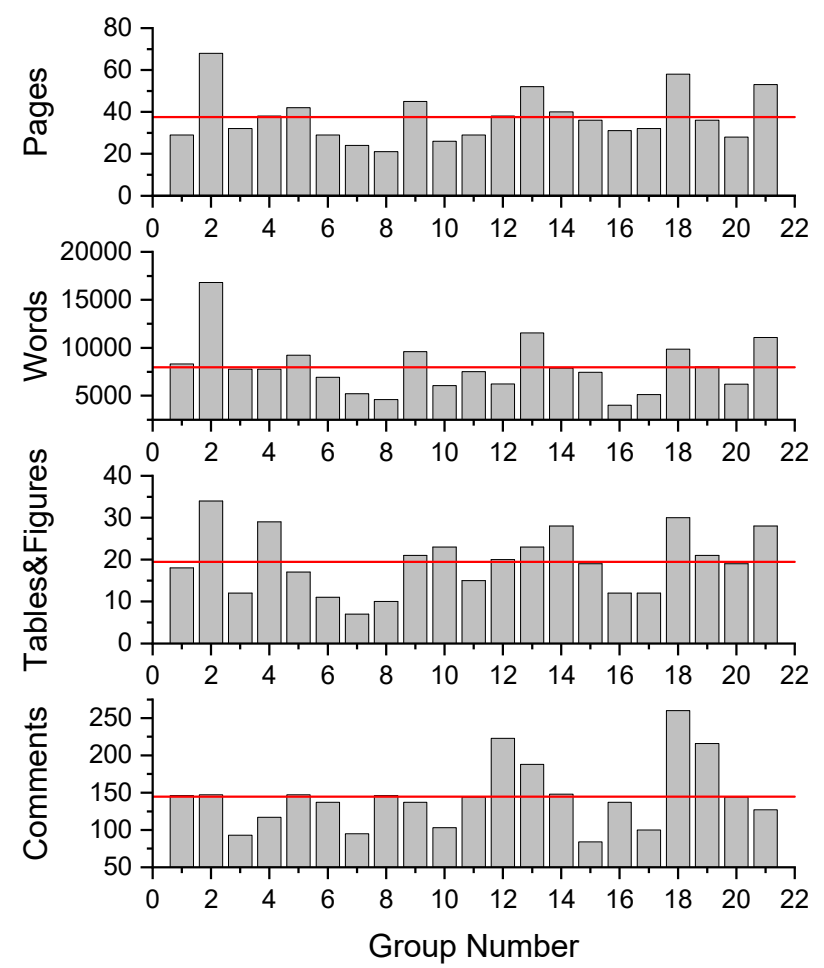

Fig. 2: The report descriptors for each group. The red line represents the average.

To quantify the level of severity the following numerical scale was given; Major error: 2 , minor error: 1 , neutral/general comment: 0 , above expectations: -2 . Due to the large variation in report lengths, the number of errors was normalized to errors per page. The errors per page could be compared to four different scenarios in the marking rubric shown in Figure 1:

1. At the intersection of a section and competency (i.e., one cell).

2. The summation of one section (i.e., one row)

3. The summation of one competency (i.e., one column).

4. The entire overall report (i.e., all cells)

For the current rubric, based on the four different scenarios, there would be 48 different permutations that could be investigated. For the purpose of this paper, only the results and discussion (R\&D) section and the technical knowledge (TK) competency parts will be investigated.
The other sections and competencies showed similar results.

For the intersection between a section and competency, there were very little correlation between the mark assigned and the number of errors per page (Figure 3). This shows the discrepancy associated with the subjective nature of some rubrics. For example, one report received a mark of marginal on the TK in the R\&D section, with only approximately 0.9 errors per page compared to another group that almost had twice as many errors but received a mark of good. Ideally, the graph below should have a decrease stepwise trend.

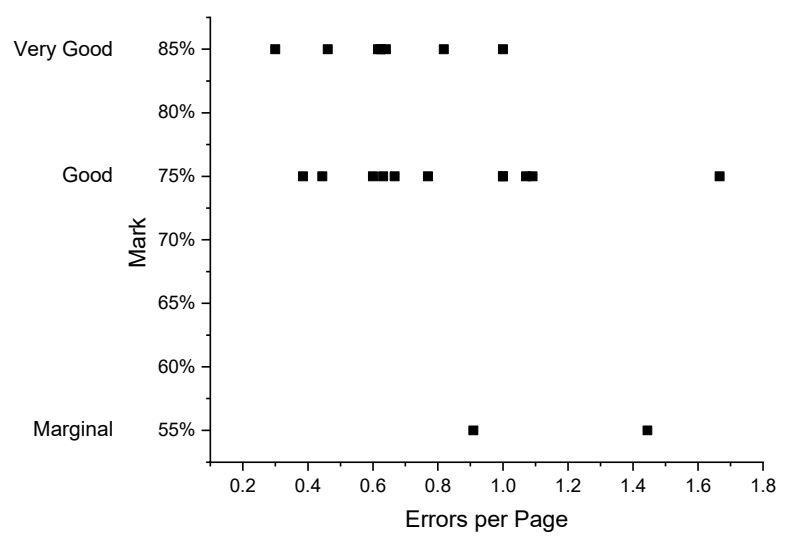

Fig. 3: The comparison between the errors per page and the mark assigned for the technical knowledge in the results and discussion section.

All seven competencies in the $R \& D$ section were compared to the errors within that section (Figure 4). From the graph it can be seen there is a downward correlation between the R\&D mark and the errors albeit not a very strong correlation.

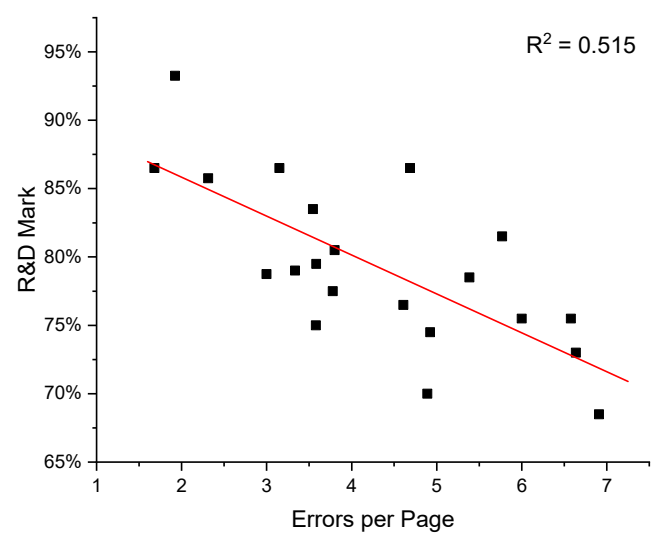

Fig.4: The comparison of errors per page against the results and discussion section overall mark.

The reverse can also be compared, where each competency is evaluated over the entire report. The mark of the technical knowledge over the whole report is 
shown in Figure 5 and a similar trend was observed as the R\&D section but with even less correlation.

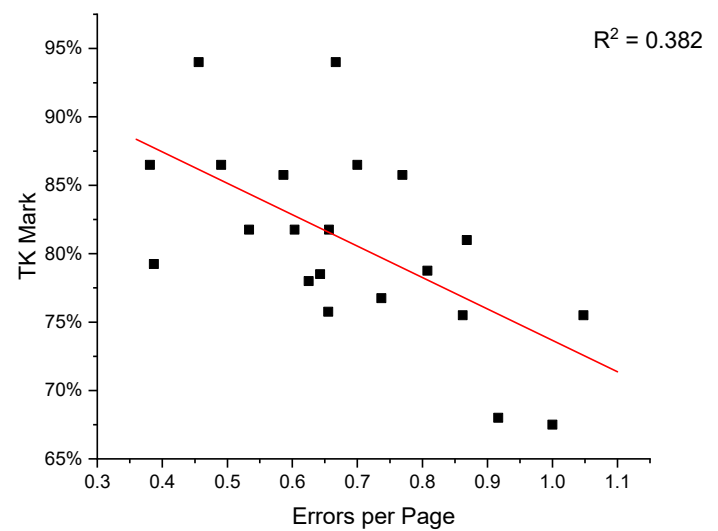

Fig. 5: The comparison of errors per page against the technical knowledge competency overall mark.

Finally, the overall mark can also be compared to all errors in the document (Figure 6). As before, a noticeable downward trend is observed with poor correlation. The weak correlation is likely cause by the subjective nature when assigning the evaluation to each cell. It can be argued that the coefficient of determination $\left(\mathrm{R}^{2}\right)$ may be adequate for this study due to the subjective nature of the evaluation. However, $\mathrm{R}^{2}$ can likely be increased if a more objective approach is taken for evaluation of each cell. A more objective approach would be to assign the marking level (e.g., Excellent) to a numerical bin based on the number of errors per page for each competency and section. This level of objectivity would then propagate throughout all other scenarios and lead to a more fair and consistent mark.

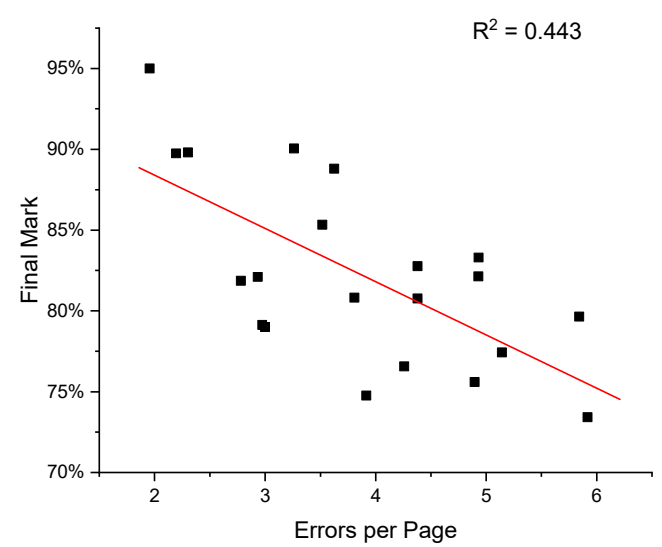

Fig 6. The comparison of errors per page against the all competencies and sections.

\section{CONCLUSIONS AND FUTURE WORK}

This preliminary work has shown, that despite the use of a specific rubric and marking criteria, the consistency of the assigned grades against the number of errors detected is somewhat lacking. Although there was some correlation between the final mark and the average number of errors per page, future improvements and work can be completed. For example, it would be useful to employ the same marking structure to different courses and different evaluators. This would first increase the amount of data available and investigate the impact of different evaluators. With the larger dataset, it be would possible to develop a model that would aid in subsequently assigning the category for each intersection in the rubric. This would create a more objective and fair evaluation of a report.

\section{Acknowledgements}

The author acknowledges with gratitude the support of the Department of Chemical and Biological Engineering for the motivation of this work.

\section{References}

[1] A. Jonsson, G. Svingby, "The use of scoring rubrics: Reliability, validity and educational consequences," Educational Research Review, vol. 2, pp. 130-144, 2007.

[2] A.R. Rezaei, and M. Lovorn, "Reliability and validity of rubrics for assessment through writing," Assessing. Writing, vol. 15, pp. 18-39, 2010.

[3] E. Panadero, and M. Romero, "To rubric or not to rubric? The effects of self-assessment on selfregulation, performance and self-efficacy," Assessment in Education: Principles, Policy and Practice, vol. 21, no. 2, pp. 133-148, 2014.

[4] E. Panadero, and A. Jonsson, "The use of scoring rubrics for formative assessment purposes revisited: A review," Educational Research Review, vol. 9, pp. 129-144, 2013

[5] J. Hudson, S. Bloxham, B. den Outer, and M. Price, "Conceptual acrobatics: talking about assessment standards in the transparency era" Studies in Higher Education, vol. 42, no. 7, pp. 1309-1323, 2017.

[6] J.Tai, R. Ajjawi, D. Boud, P. Dawson, E. Panadero, "Developing evaluative judgement: enabling students to make decisions about the quality of work," Higher Education, vo. 76, no. 3, pp. 467-481, 2018. 